\section{Evaluation and identification of histamine-forming bacteria on fish products of middle Adriatic Sea}

\author{
Claudia Costanza, ${ }^{1}$ Matilde Cecchini, ${ }^{1}$ \\ Rocco Mancusi, ${ }^{1}$ Alcide Mosso, ${ }^{2}$ \\ Gabriele Giani, ${ }^{3}$ Roberto Rosmini, \\ Marcello Trevisani \\ 'Dipartimento di Scienze Mediche \\ Veterinarie, Università degli Studi \\ di Bologna - Alma Mater Studiorum, \\ Ozzano dell'Emilia; 'Dipartimento di \\ Sanità Pubblica AUSL Ferrara, Servizio \\ Veterinario, Ferrara; ${ }^{3}$ Dipartimento di \\ Sanità Pubblica AUSL Imola, Servizio \\ Veterinario, Imola, Italy
}

\section{Abstract}

Regulation EU 2073/2005 sets maximum concentration for histamine in fish and products thereof. To meet these criteria, manufacturers have to define performance objectives, such as the maximum allowed prevalence and number/activity of histamine-producing bacteria at relevant stage of production. In order to assess the presence and decarboxylase activity of contaminant bacteria we examined 51 samples of blue fish caught and processed in Emilia Romagna region. We collected 50 gr of fish (skin and gills or the entire product) from 10 sample units from every lot. Analytical samples were cultured in Trypticase Soy Broth supplemented with histidine and pyridoxal $\mathrm{HCl}$. Histamine was measured with an electrochemical biosensor after incubation at both $37^{\circ} \mathrm{C}$ for $24 \mathrm{~h}$ and $18-22^{\circ} \mathrm{C}$ for $48 \mathrm{~h}$. Enrichments that showed relevant enzymatic activity were seeded on Niven agar to isolate suspected colonies and DNA extracts from these bacteria were analyzed by polymerase chain reaction (PCR) for detecting specific sequences of the gene encoding pyridoxaldependent histidine decarboxylase (HDC). Overall, 29.4\% samples showed relevant production of histamine in broth cultures (above a cut-off value set at $250 \mathrm{ng} / \mathrm{mL}$ ) and $53.3 \%$ of them (8 out of 15 samples) allowed detection of HDC positive strains. All of them were typed as Morganella, which appears to be the most common of fish caught in middle Adriatic sea. Ten out of the twelve positive samples with enrichment cultures incubated at both 37 and $18-22^{\circ} \mathrm{C}(83 \%)$ showed higher decarboxylase activity at room temperature, suggesting the presence of psychrotolerant strains. In addition, the prevalence of histamine-producing bacteria was higher at retail than at production level, probably as a consequence manipula- tions and cross-contamination. The risk correlated to development of histamine-producing psychrotolerans bacteria cannot be controlled only with storage temperature: it is necessary for the food business operators to evaluate specific performance objectives, define useful parameters to qualify suppliers, and guarantee microbiological criteria fixed by Regulation EU 2073/2005

\section{Introduzione}

L'intossicazione da istamina si manifesta in conseguenza dell'assunzione di prodotti ittici nei quali l'ammina biogena si accumula, per effetto dell'attività di batteri che utilizzano l'istidina libera, presente in concentrazione rilevante in talune specie ittiche descritte nel Regolamento 853/2004 (Commissione Europea, 2004). Il Regolamento CE 2073/2005 (Commissione Europea, 2005) definisce criteri di accettabilità sulla concentrazione d'istamina presente nei prodotti della pesca fissandone i limiti a $100 \mathrm{mg} / \mathrm{kg} \mathrm{M}$ con una tolleranza fino a $200 \mathrm{mg} / \mathrm{kg} \mathrm{M}$ in non più di due unità campionarie $(\mathrm{N}=9)$ sul prodotto fresco. Per soddisfare tali criteri i produttori devono definire degli obiettivi di performance, quali la prevalenza e l'attività dei batteri produttori d'istamina in fasi rilevanti della filiera produttiva. I prodotti ittici a rischio sono perciò oggetto di controlli da parte delle autorità sanitarie e gli operatori del settore ittico devono essere a conoscenza della qualità microbiologica dei prodotti lavorati e posti da loro in commercio. I batteri istaminogeni sono stati classificati, in base all'attività enzimatica, in deboli, medi e forti produttori (Bjornsdottir et al., 2010); questi ultimi sono capaci di produrre quantità d'istamina superiori a $1000 \mathrm{ng} / \mathrm{mL}$ in brodocoltura. La maggior parte dei batteri forti produttori che si osservano nel pesce fresco e nei prodotti ittici appartengono alla famiglia delle Enterobacteriaceae (Morganella, Raoultella, Erwinia, Proteus e altri) (Cantoni, 2008). Anche i batteri del genere Photobacterium (P. damsealae e P. phosphoreum) mostrano forte attività istaminogena $\mathrm{e}$ sono stati spesso implicati in casi clinici d'intossicazione (Emborg e Dalgaard, 2006; Landete et al., 2008). Poiché i prodotti ittici sono normalmente conservati a temperature prossime $\mathrm{a}+0^{\circ} \mathrm{C}$, assumono un maggior significato le specie e i biotipi psicrotolleranti. Obiettivo di questo studio è stato quello di valutare la prevalenza e l'attività dei batteri produttori d'istamina in pesci e prodotti ittici di specie a rischio, catturati e lavorati nel medio Adriatico, seguendone l'evoluzione nel corso della filiera, dalla fase di sbarco fino alla commercializzazione al dettaglio.
Correspondence: Marcello Trevisani, Dipartimento di Scienze Mediche Veterinarie, Università degli Studi di Bologna - Alma Mater Studiorum, via del Florio 2, 40064 Ozzano dell'Emilia, Italy.

Tel. +39.051.2097330 - Fax: +39.051.2097346.

E-mail: marcello.trevisani@unibo.it

Key words: Morganella morganii, Morganella psychrotolerans, Histidine decarboxylase, Histamine, Shelf life, Fish poisoning.

Conflict of interests: the authors declare no potential conflict of interests.

Received for publication: 15 January 2013.

Revision received: 7 March 2013.

Accepted for publication: 7 March 2013.

This work is licensed under a Creative Commons Attribution 3.0 License (by-nc 3.0).

(C) Copyright C. Costanza et al., 2013

Licensee PAGEPress, Italy

Italian Journal of Food Safety 2013; 2:e7

doi:10.4081/ijfs.2013.e7

\section{Materiali e Metodi}

Nel periodo Novembre 2011-Aprile 2012 sono stati analizzati 51 campioni provenienti da pescherecci, impianti di lavorazione e pescherie. Le specie oggetto di campionamento sono state alici (17 campioni), sarde (9 campioni), sgombri (9 campioni), tonno (8 campioni) aringhe (2 campioni), lanzardi, alacce e palamite (1 campione). Il campionamento è stato ripetuto due volte presso ciascun operatore (Tabella 1). Il prodotto fresco prelevato allo sbarco e in pescheria era di origine nazionale e faceva parte della stessa filiera ittica, mentre la materia prima dei prodotti trasformati seguiva percorsi differenti e aveva origini sia nazionale sia internazionale (Irlanda del Nord, Atlantico, Atlantico centroorientale e oceano Pacifico). In parallelo al campionamento è stata condotta un'indagine per raccogliere dati sulle condizioni igieniche delle attività produttive e distributive e sui processi di lavorazione. I campioni sono stati trasportati in laboratorio refrigerati e, nel caso dei pesci freschi, analizzati entro sei ore dal prelievo. Da ogni campione, formato da 10 unità di prodotto, sono stati prelevati $50 \mathrm{~g}$ di materiale (branchie e cute per i prodotti freschi); questi sono stati diluiti in proporzione di 1:5 in phosphate buffered saline (PBS) e poi passati in Stomacher ${ }^{\circledR}$ per $3 \mathrm{~min}$. Un mL dell'omogenato è stato diluito in $9 \mathrm{~mL}$ di brodo di arricchimento tryptic soy broth (TSB) contenente istidina (1\%) e piridoxal $\mathrm{HCl}(0,0005 \%)$. 
Per tutti i campioni è stato fatto un arricchimento a $37^{\circ} \mathrm{C}$ per 24 ore. Al fine di rilevare anche l'attività decarbossilasica dei batteri Gram negativi psicrotolleranti e alofili, nel secondo campionamento (campioni da 24 a 51) è stata utilizzata anche una formulazione modificata del terreno contenente $\mathrm{NaCl}(2 \%)$ con incubazione a $18-22^{\circ} \mathrm{C}$ per 48 ore. Gli arricchimenti sono stati poi analizzati mediante un biosensore elettrochimico per misurare la quantità d'istamina prodotta in coltura. Tale strumento misura la corrente prodotta dall'ossidazione del perossido d'idrogeno che è generato da un sistema bi-enzimatico (diaminossidasi e perossidasi) immobilizzato sulla superficie di un elettrodo (Trevisani, comunicazione personale). È stato predefinito un valore di cutoff pari a $250 \mathrm{ng} / \mathrm{mL}$ di arricchimento (50 ng/g di tessuto) e tutti i campioni che mostravano attività enzimatica superiore sono stati seminati su terreno di Niven modificato (Mavromatis and e Quantick, 2002) e incubati a temperatura ambiente fino a un massimo di quattro giorni. Da ogni piastra di Niven sospetta per la presenza di batteri produttori d'istamina, ovvero con la presenza di colonie contornate da alone viola, sono state prelevate fino a 50 colonie per il test polymerase chain reaction (PCR), atto a rilevare la presenza nel genoma batterico di sequenze specifiche del gene istidina decarbossilasi (HDC) piridoxal dipendente (Landete et al., 2008). A tal fine sono stati utilizzati il protocollo e i primer (HIS2F e HIS2R) individuati da de las Rivas et al. (2006), in grado di generare un prodotto di amplificazione di 531 bp anche da diversi batteri, tra cui quelli dei generi Morganella e Photobacterium. Le colonie che mostravano prodotti di amplificazione specifici sono state sottoposte a caratterizzazione biochimica tramite test API20E.

\section{Risultati}

Quindici dei 51 campioni analizzati (29,4\%) hanno mostrato positività per la produzione di istamina nelle colture di arricchimento batterico (Tabella 1). La prevalenza è stata del 20 $50 \%$ in alici e sarde campionate allo sbarco e del $71-80 \%$ nelle stesse specie campionate in pescheria. La presenza di attività istidinodecarbossilasica batterica è stata rilevata anche in altri 12 campioni $(23,5 \%)$ ma con valori $<250 \mathrm{ng} / \mathrm{mL}$. Riguardo alle altre specie campionate è da annotare la positività di palamite e aringhe prelevate in pescheria e la negatività di lanzardi e sgombri prelevati allo sbarco. Le colture di arricchimento batterico dei prodotti campionati in fase di lavorazione (sgombri e tranci di tonno congelati, sgombri e alici marinate e sott'olio, tonno sterilizzato, pasta di alici, alici salate, tonno affumicato e sughi di tonno) non hanno mai fatto rilevare la presenza di istidino-decarbossilasi. Una percentuale elevata (83\%) dei campioni positivi ha evidenziato un'attività istamino-decarbossilasica maggiore a temperatura ambiente (Tabella 2). Dodici dei 15 campioni nettamente positivi ( $>250 \mathrm{ng} / \mathrm{mL}$ istamina prodotta in brodocoltura) hanno fatto rilevare la presenza di colonie sospette nelle colture su terreno di Niven. Il DNA batterico estratto dalle colonie sospette isolate relative a 8 campioni ha mostrato la presenza di sequenze specifiche del gene HDC. I batteri positivi per il genotipo HDC sono stati riconosciuti mediante caratterizzazione morfologica e biochimica (test API20E). I batteri erano infatti bastoncelli Gram-negativi, fermentanti il glucosio, mobili, ossidasi negativi e catalasi positivi, fenilalanina deaminasi positivi, gelatina negativi, ureasi positivi, indolo positivi e citrati negativi.

\section{Discussione}

La presenza di batteri produttori d'istamina è stata rilevata in molti $(29,4 \%)$ dei campioni di pesce prelevati. Seguendo alcuni prodotti ittici lungo la filiera (es. alici e sarde) si è notato che la prevalenza della contaminazione, già elevata allo sbarco (20-50\%) risultava aumentata nei campioni prelevati presso le pescherie (71-80\%). La microflora istaminogena era prevalentemente costituita da batteri psicrotolleranti visto che in una percentuale elevata (83\%) dei campioni positivi l'attività decarbossilasica era maggiore nelle colture di arricchimento batterico incubate a temperatura ambiente che in quelle a $37^{\circ} \mathrm{C}$. Anche se da 12 dei 15 campioni positivi è stato possibile isolare colonie sospette su terreno di Niven (l'alcalinizzazione è indice della produzione di amine), solo in 8 casi ( $67 \%$ degli isolati) è stata individuata la presenza di sequenze specifiche del gene HDC piridoxal dipendente. In tutti questi casi la caratterizzazione morfologica e biochimica ha permesso il riconoscimento del genere Morganella. Riteniamo che la

Tabella 1. Prevalenza e attività dei batteri produttori d'istamina in diverse fasi e tipologie di prodotti ittici.

\begin{tabular}{|c|c|c|c|c|c|c|}
\hline Fase & Numero di campioni & Tipologia & Specie & \multicolumn{3}{|c|}{ Batteri produttori d'istamina negli arricchimenti } \\
\hline Sbarco & 11 & Fresco & $\begin{array}{l}\text { Sarda } \\
\text { Alice } \\
\text { Lanzardo } \\
\text { Sgombro }\end{array}$ & $\begin{array}{l}2 / 4 \\
1 / 5 \\
0 / 1 \\
0 / 1\end{array}$ & $\begin{array}{l}(50) \\
(20) \\
(0) \\
(0)\end{array}$ & $\begin{array}{c}381-5736 \\
9943 \\
<250 \\
<250\end{array}$ \\
\hline Pescheria & 17 & Fresco & $\begin{array}{l}\text { Sarda } \\
\text { Alice } \\
\text { Aringa } \\
\text { Palamita }\end{array}$ & $\begin{array}{l}5 / 6 \\
5 / 7 \\
1 / 2 \\
1 / 1\end{array}$ & $\begin{array}{l}(83) \\
(71) \\
(50) \\
(100)\end{array}$ & $\begin{array}{c}281-5097 \\
444-5097 \\
3691 \\
302\end{array}$ \\
\hline Lavorazione & 23 & $\begin{array}{l}\text { Congelato } \\
\text { Sotto sale } \\
\text { Pasta } \\
\text { Marinato } \\
\text { Sott'olio } \\
\text { Sterilizzato } \\
\text { Affumicato a freddo } \\
\text { Sugo pastorizzato } \\
\text { Sugo non pastorizzato }\end{array}$ & $\begin{array}{l}\text { Sgombro } \\
\text { Tonno } \\
\text { Alice } \\
\text { Alice } \\
\text { Sgombro } \\
\text { Alice } \\
\text { Sgombro } \\
\text { Tonno } \\
\text { Tonno } \\
\text { Tonno } \\
\text { Tonno }\end{array}$ & $\begin{array}{l}0 / 3 \\
0 / 2 \\
0 / 2 \\
0 / 3 \\
0 / 1 \\
0 / 2 \\
0 / 4 \\
0 / 1 \\
0 / 2 \\
0 / 2 \\
0 / 1\end{array}$ & $\begin{array}{l}(0) \\
(0) \\
(0) \\
(0) \\
(0) \\
(0) \\
(0) \\
(0) \\
(0) \\
(0) \\
(0)\end{array}$ & $\begin{array}{l}\mathrm{nr} \\
\mathrm{nr} \\
\mathrm{nr} \\
\mathrm{nr} \\
\mathrm{nr} \\
\mathrm{nr} \\
\mathrm{nr} \\
\mathrm{nr} \\
\mathrm{nr} \\
\mathrm{nr} \\
\mathrm{nr}\end{array}$ \\
\hline
\end{tabular}

Nr, non rilevabile. 
Tabella 2. Attività dei batteri produttori d'istamina nei campioni positivi e concentrazione d'istamina rilevata a differenti temperature d'incubazione.

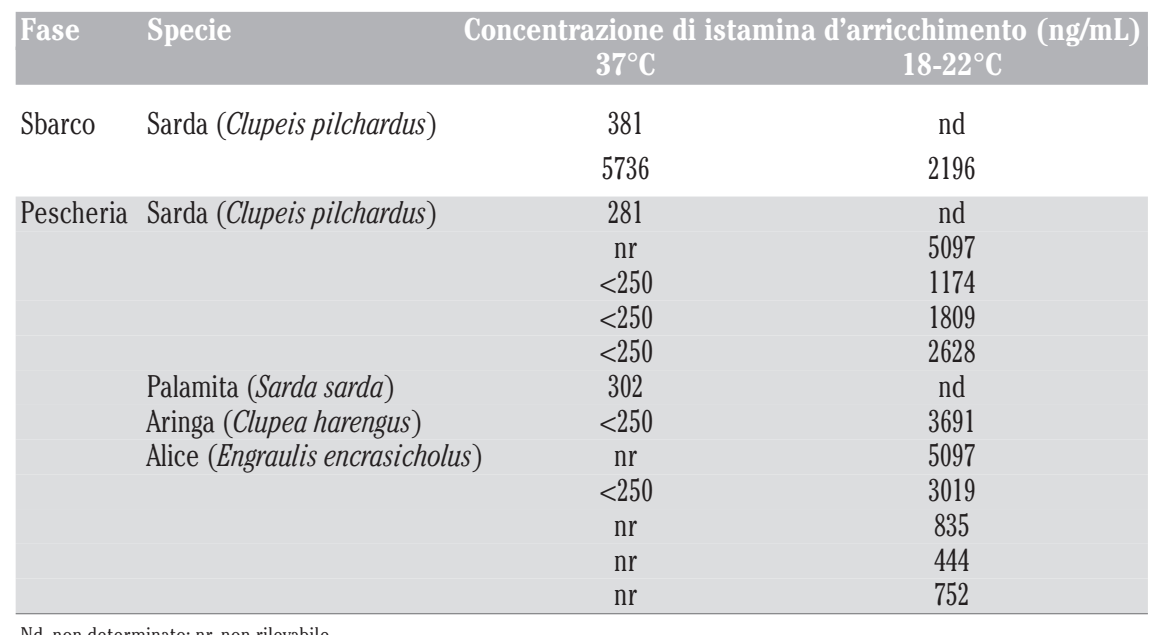

$\mathrm{Nd}$, non determinato; nr, non rilevabile.

discrepanza tra le positività rilevate tramite il rilievo dell'attività istidino-decarbossilasica nelle brodocolture di arricchimento, la presenza di colonie sospette sul terreno di Niven e la conferma PCR degli isolati dipenda dal fatto che sullo stesso terreno possono assumere colorazione viola anche colonie appartenenti a specie non produttrici d'istamina (LopezSabater et al., 1996; Fletcher et al., 1998; Bjornsdottir et al., 2009) e dalla differente capacità delle specie microbiche istaminogene di sviluppare sul terreno di Niven (falsi negativi). Non è stato possibile ottenere isolati positivi al test PCR da tutti i campioni che hanno mostrato un'attività istaminogena rilevante (>250 ng/mL) nelle brodocolture di arricchimento. Bjornsdottir et al. (2010) ritengono che falsi negativi derivino dall'acidità del terreno di Niven (pH 5,3), la quale può inibire lo sviluppo di alcuni ceppi batterici produttori d'istamina. È anche da annotare che basse concentrazioni d'istamina nelle brodocolture di arricchimento possono essere rilevate anche in assenza di batteri produttori a causa dell'attività enzimatica residua di batteri non più vitali (Kanki et al., 2007) 0 ad attività di altre decarbossilasi non specifiche (Bjornsdottir et al., 2010). Il fatto che nel nostro studio sia stata evidenziata la prevalenza assoluta del genere Morganella fa supporre che questa sia la specie forte produttrice d'istamina maggiormente presente nel medio Adriatico; non si può però escludere che l'impiego del terreno differenziale di Niven abbia limitato la sensibilità del metodo e la possibilità d'isolamento. Ad avvalorare questa ipotesi è stata la presenza di un campione di alici positivo al test PCR su pool di colonie che ha perso vitalità nei successivi passaggi di purificazione. Pur mostrando un'alta percentuale di falsi positivi, il Niven rimane un terreno vantaggioso poiché è l'uni- co che permette una discriminazione dei batteri sulla base della capacità di alcalinizzare il substrato liberando istamina nello stesso. Il rilievo di una maggiore attività istaminogena negli arricchimenti a temperatura ambiente (11/12 campioni tra quelli esaminati sia a $+37^{\circ} \mathrm{C}$ che $\mathrm{a}+18-22^{\circ} \mathrm{C}$ ) è indicativa della presenza di batteri psicrotolleranti. Emborg et al. (2006) hanno riconosciuto l'esistenza di una specie distinta nell'ambito del genere Morganella, da loro denominata M. psychrotolerans, la cui capacità di moltiplicare e produrre forti quantità d'istamina nel muscolo di pesce conservato a condizioni di refrigerazione la rende particolarmente pericolosa, in modo particolare per i prodotti ittici refrigerati a conservazione prolungata e stabilizzati sottovuoto o per mezzo di atmosfere modificate. La prevalenza della flora istaminogena nei lotti di alici è passata dal $20 \%$ nei prodotti campionati allo sbarco al $71 \%$ in quelli prelevati in pescheria, provenienti dalla stessa filiera, mentre in quelli di sarde dal 50 all'83\%. Le operazioni di trasferimento, cernita e aggiunta di ghiaccio, osservate durante l'indagine eseguita in parallelo ai campionamenti, possono comportare un aumento della prevalenza delle contaminazioni, come rilevato anche da altri autori che ritengono di conseguenza di fondamentale importanza la cura dell'igiene personale, delle lavorazioni e le operazioni di sanificazione delle attrezzature e dell'ambiente, al fine di limitare lo sviluppo dei batteri produttori d'istamina (Kimata, 1961; Yoshinaga and e Frank, 1982). Anche le manipolazioni e il metodo di pesca rivestono un ruolo importante nei confronti del pericolo istamina: come affermano gli autori delle linee guida FDA (2011) tutto ciò che provoca lesioni al pesce azzurro può favorire il passaggio di batteri all'interno delle masse muscolari di quest'ultimo, portan- do a contatto flora microbica e istidina libera e facilitando quindi la produzione d'istamina.

\section{Conclusioni}

In conclusione, dobbiamo rilevare che l'alta prevalenza di batteri forti produttori d'istamina già allo sbarco rende auspicabile un controllo per definire la qualità della materia prima di deciderne la destinazione d'uso e la durata della vita commerciale, nel rispetto dei criteri microbiologici imposti dalla normativa vigente. Il metodo di analisi utilizzato in questo studio permette di valutare rapidamente l'attività istaminogena della flora microbica contaminante. La classificazione dei lotti di prodotto in base a tale parametro può essere utilizzata dagli operatori del settore che operano nella commercializzazione e trasformazione di prodotti ittici a rischio (pesci ricchi d'istidina libera) per definire in modo appropriato la durata della vita commerciale da attribuire a questi prodotti. Ciò richiede tuttavia anche una valutazione del potenziale di crescita delle specie produttrici d'istamina. Le specie batteriche prevalenti possono variare in funzione delle specie ittiche, delle zone di pesca e delle tecniche di pesca. Lo sviluppo di modelli di stima del rischio richiederà pertanto ulteriori studi.

\section{Bibliografia}

Bjornsdottir BK, Bolton GE, Jaykus LA, Green PDM, Green DP, 2010. Development of molecular-based methods for determination of high histamine producing bacteria in fish. Int J Food Microbiol 139:161-7.

Bjornsdottir BK, Bolton GE, McClellan-Green PD, Jaykus L-E, Green DP, 2009. Detection of gram-negative histamine - producing bacteria in fish: a comparative study. $\mathrm{J}$ Food Protect 72:1987-91.

Cantoni C, 2008. Sistematica dei batteri produttori di istamina nelle carni di pesce. Il Pesce 6:115-19.

Commissione Europea, 2004. Regolamento del parlamento europeo e del consiglio del 29 aprile 2004 che stabilisce norme specifiche in materia di igiene per gli alimenti di origine animale, 853/2004/CE. In: Gazzetta Ufficiale, L 139/55, 30/04/2004.

Commissione Europea, 2005. Regolamento del parlamento europeo del 15 novembre 2005 sui criteri microbiologici applicabili ai prodotti alimentari, 2073/2005/CE. In: Gazzetta Ufficiale, L338/1, 22/12/2005.

de las Rivas B, Macrobal A, Carrascosa AV, Munoz R, 2006. PCR detection of foodborne bacteria producing the biogenic amines 
histamine, tyramine, putrescine, and cadaverine. J Food Protect 69:2509-14.

Emborg J, Dalgaard P, 2006. Formation of histamine and biogenic amines in coldsmoked tuna: an investigation of psychrotolerant bacteria from samples implicated in cases of histamine fish poisoning. $\mathrm{J}$ Food Protect 69:897-906.

Emborg J, Dalgaard P, Ahrens P, 2006. Morganella psychrotolerans sp. nov., a histamine-producing bacterium isolated from various seafood. Int J Syst Evol Micr 56:2473-9.

FDA, 2011. Fish and fishery products hazards and controls guidance. Food and Drug Administration ed., Silver Spring, MD, USA. Disponibile al sito: http://www.fda.gov/Food/GuidanceRegulati on/GuidanceDocumentsRegulatoryInform ation/Seafood/ucm2018426.htm

Fletcher GC, Summers G, Van Veghel PWC, 1998. Levels of histamine and histamineproducing bacteria in smoked fish from New Zealand markets. J Food Protect
61:1064-70.

Kaneko JJ, Bell JW, Hawn DR, 2004. Verification of a HACCP-based strategy for the control of histamine for the fresh tuna industry. PacMar Inc. ed., Honolulu, HI, USA.

Kanki M, Yoda T, Tsukamoto T, Baba E, 2007. Histidine decarboxylases and their role in accumulation of histamine in tuna and dried saury. Appl Environ Microb 73:146773.

Kimata M, 1961. The histamine problem. In: Borgstrom G, ed. Fish and food: production, biochemistry and microbiology. Academic press, New York, NY, pp. 329-52.

Landete JM, de las Rivas B, Marcobal A, Munoz $\mathrm{R}, 2008$. Updated molecular knowledge about histamine biosynthesis by bacteria. Crit Rev Food Sci 48:697-714.

Lopez-Sabater EI, Rodriguez-Jerez JJ, Hernandez-Herrero M, Mora-Ventura MT, 1996. Incidence of histamine-forming bacteria and histamine content in scombroid fish species from retail markets in the
Barcelona area. Int J Food Microbiol 28:411-8.

Mavromatis P, Quantick PC, 2002 Modification of Niven's medium for the enumeration of histamine- forming bacteria and discussion of the parameters associated with its use. J Food Protect 65:546-51.

Takahashi H, Kimura B, Yoshikawa M, Fujii T, 2003. Cloning and sequencing of the histidine decarboxylase genes of gram-negative, histamine-producing bacteria and their application in detection and identification of these organisms in fish. Appl Environ Microb 69:2568-79.

Takahashi H, Sato M, Kimura B, Ishikawa T, Fujii T, 2007. Evaluation of PCR-singlestrand conformational polymorphism analysis for identification of gram-negative histamine-producing bacteria isolated from fish. J Food Protect 70:1200-05.

Yoshinaga DH, Frank HA, 1982. Histamine: producing bacteria in decomposing skipjack tuna (Katsuwonas pelams). Appl Environ Microb 44:447-52. 